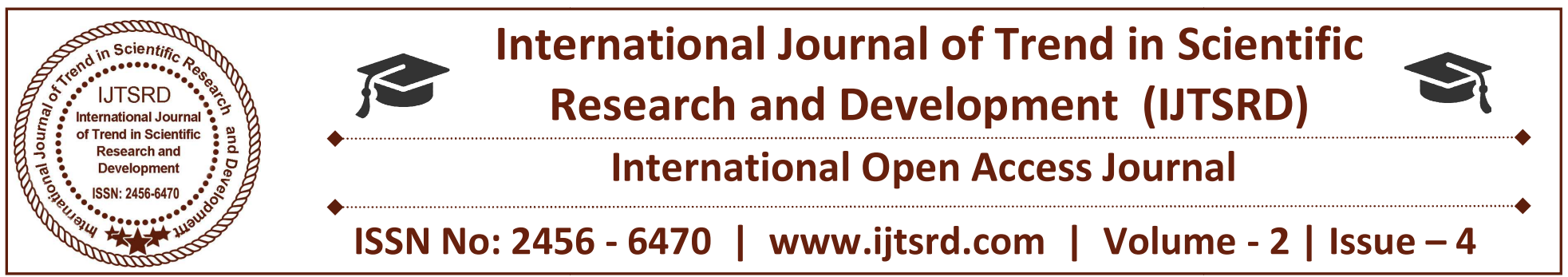

\title{
Comparison of Various Waveform Contenders of 5G Wireless Communication Based on OFDM
}

\author{
Raksha Vishnoi ${ }^{1}$, Saurabh Gaur ${ }^{2}$, Ashish Verma ${ }^{2}$ \\ ${ }^{1}$ PG Student , ${ }^{2}$ Associate Professor \\ ECE Department, M.I.T., Ujjain, Madhya Pradesh, India
}

\begin{abstract}
This paper presents an extensive and fair comparison among the most promising waveform contenders for the $5 \mathrm{G}$ air interface which includes- Filtered OFDM, Filter-bank multi carrier (FBMC), universal filtered multi-carrier (UFMC) has been compared with OFDM in terms of spectral efficiency and bit error rate using mat lab. The disadvantages of OFDM have been addressed and it has shown that (fofdm), (UFMC), (FBMC) could be a more effective solution. FBMC is a method for improving out of band (OOB) characteristics by filtering each subcarrier, it is also expected to improve the Inter-Carrier Interference (ICI) characteristics while UFMC is a method for improving $\mathrm{OOB}$ characteristics by filtering each block.
\end{abstract}

Keywords: FBMC, UFMC, ICI, KPIs and $O O B$

\section{INTRODUCTION}

The evolution of internet and the growing demand of high data rate users have given Dynamic spectrum access networks a widespread attention in the recent years. orthogonal frequency division multiplexing (OFDM) based on multi-carrier has high-power outof-band (OOB).This characteristic causes adjacent channel interference (ACI). OFDM uses a wide guard band in order to avoid ACI. It decreases spectral efficiency when a number of mobile devices simultaneously access a base station. Next generation mobile communication system requires high-level key performance indicators (KPIs). It is difficult for OFDM to satisfy the KPIs. Mobile subscribers are demanding an enormous increase of the data rates and the capacity is to be increased up to 1,000 times within the next 5 years.
Several new waveforms are already proposed for $5 \mathrm{G}$ : (FBMC), (UFMC) \& (f-OFDM). The main objective of all these modulations is to reduce the out of-band emissions and take advantage of those gap bands allowing narrowband communication to be carried out in the resources left unused by bandwidth [1].

\section{Orthogonal Frequency Division Multiplexing (OFDM)}

In OFDM multicarrier system, the frequency spectrum of the subcarriers is overlapped with the least frequency spacing and the orthogonality is attained amid the various subcarriers. The input stream is subdivided into parallel data streams by means of the serial to parallel (S/P) converter, that are then passed into an inverse fast Fourier transformation (IFFT) block to produce time sequence of the streams. Consequently, by totalling a cyclic prefix (CP), the OFDM symbol time sequences are extended [1],[2]. The CP is a copy of the latter portion of the symbol that is added in the start of the sequence and should be greater than the network deferral spread in order to diminish the inter symbol interference (ISI) produced by the influx of various OFDM symbols with distinct delay. The resultant digital signal is transformed into analog form and transmitted over the channel. At the receiver end, the signal is reconstructed into digital form and the far Fourier transform (FFT) is achieved in the received streams after eradicating the CP. Finally, the parallel streams are collected into a single stream as the original transmitted one. Some of the disadvantages of OFDM are enumerated below: 1. Decreased spectral efficiency owing to the $\mathrm{CP}$ employed. 


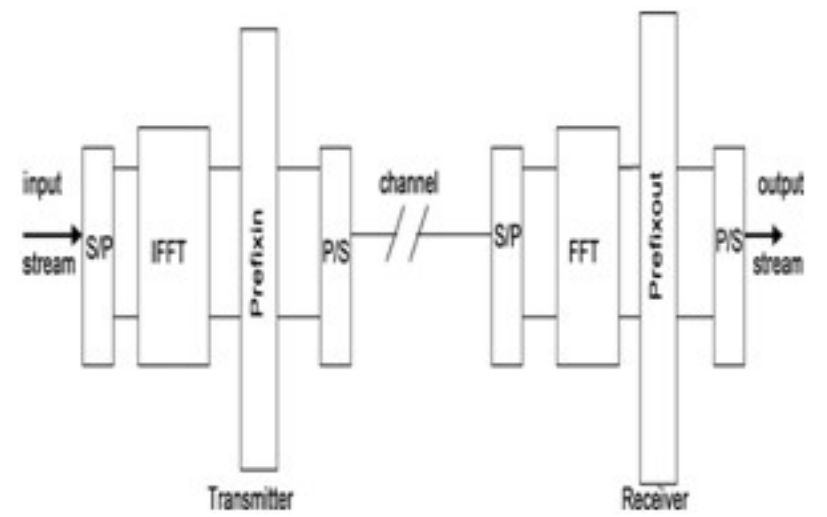

Figure 1: Block Diagram of OFDM

2. High spectral leakage owing to the rectangular windowing.

3. Interference amid the unsynchronized signal in the neighboring bands.

OFDM is a widely used modulation and multiplexing technology, which has become the basis of many telecommunications standards including wireless local area networks (LANs), digital terrestrial television (DTT) and digital radio broadcasting in the world. It ia multicarrier transmission scheme which divides the bandwidth into different sub-channels known as sub- carriers so as to multiplex in both time and frequency domain. OFDM data are generated by taking symbols in the spectral space using M-PSK, QAM, etc, and convert the spectra to time domain by taking the Inverse Discrete Fourier Transform (IDFT). Since Inverse Fast Fourier Transform (IFFT)is more cost effective to implement. It is basically a multi-carrier modulation technique [2].

\section{Filtered - OFDM (F-OFDM)}

F-OFDM is one of the proposed waveforms for the evolution towards 5G.In OFDM the effect of large out of band emission due to the rectangular shaping of temporal signals is largely alleviated in F-OFDM by using transmit filter which is cascaded after the modulator. Filtering process in f-OFDM is done through specially filters which are suitably designed to suppress high side lobes thereby making FilteredOFDM a bandwidth efficient technique. Besides this, filtering operation becomes challenging when the spectrum pulses are not continuous and the filter is redesigned for each available chunk of spectrum. Hence, we will introduce ISI in Consecutive

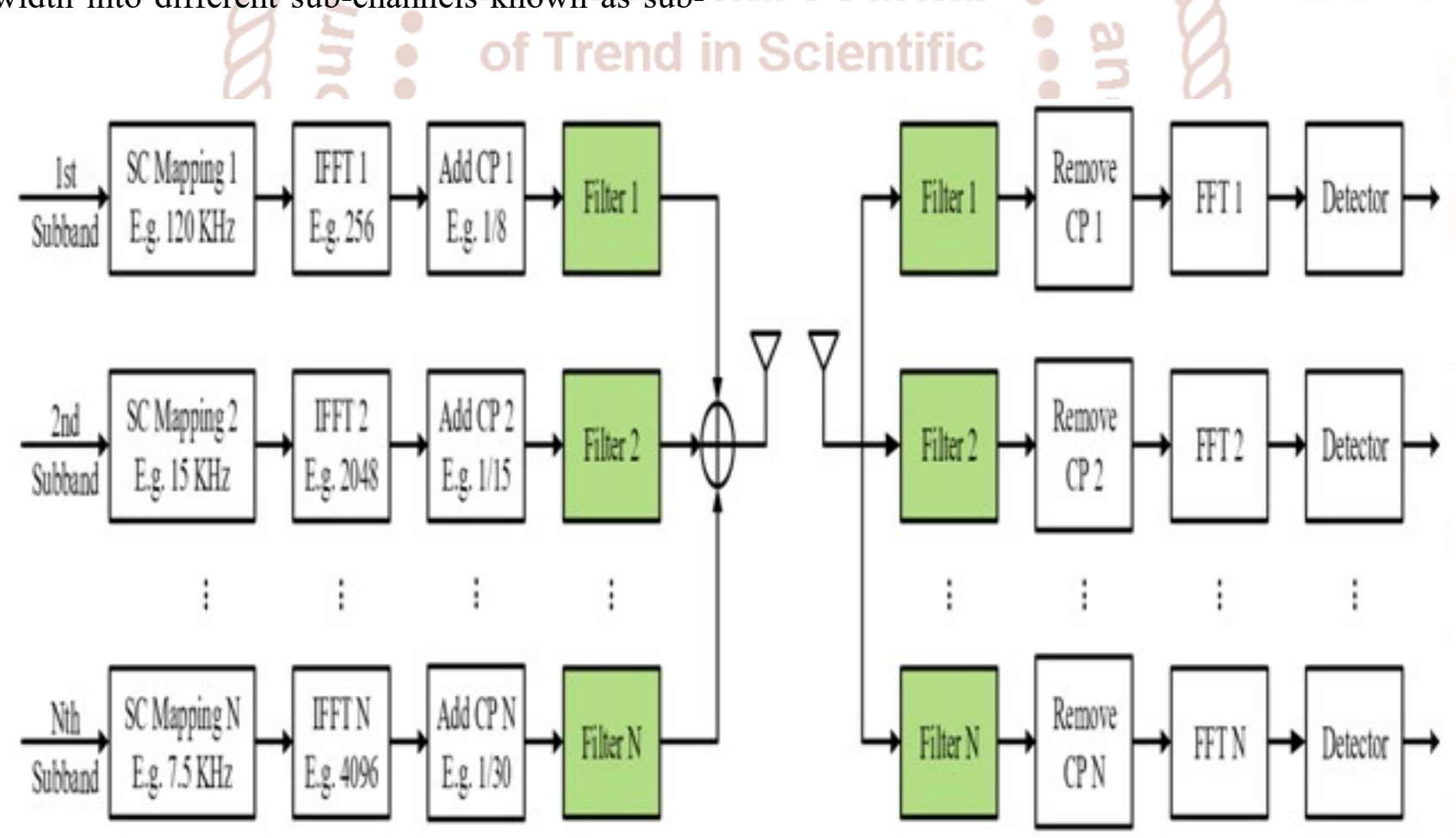

Figure 2: Block Diagram of f-ofdm Transceiver [5] 


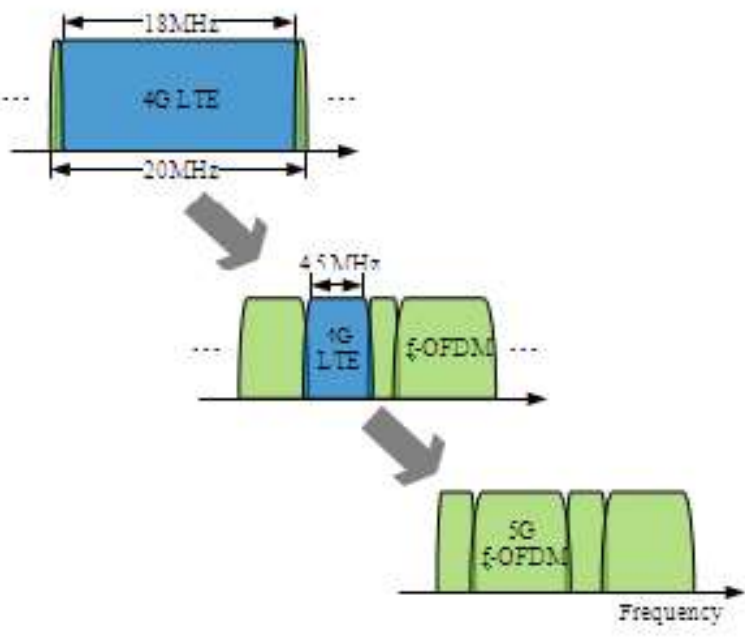

Figure 3: Possible evolution path from OFDMin4GLTEtof-OFDMin $5 \mathrm{G}$

Symbols arriving at the receiver. In order to cope with this ISI we can enlarge the Cyclic prefix (CP) so as to cancel both, the effect of multipath and filtering. Enlarging the $\mathrm{CP}$ means reducing the performance by introducing a larger overhead, so we need to avoid it. The main idea is filtering the OFDM signal due to which the out-of-band emissions will be reduced making adjacent gap bands available and easier to use for other purposes. f-OFDM provides both forward and backward compatibility. In f-OFDM the available bandwidth is divided in several sub-bands, each one having different numerology and accommodating different kind of services. Even though OFDM is considered to be spectrum efficient, still $10 \%$ of allocated BW is wasted to allow attenuation of the signal.

To achieve orthogonally both in frequency and time in OFDM, extra signalling to assure synchronization is needed, especially for uplink transmission. In this way, we brake intentionally the time-domain orthogonally between consecutive f-OFDM symbols for lower OOBE. Consequently, asynchronous transmission between sub-bands is now supported by Filtered - OFDM [2],[3].

\section{FBMC}

The FBMC technique overcomes the limitations of OFDM by adding generalized pulse shaping filters which delivers a well localized sub channel in both time and frequency domain. Consequently, FBMC systems have more spectral containment signals and offer more effective use of the radio resources where no $\mathrm{CP}$ is required. It can be seen that the filter banks on the transmitter side and the receiver side consist of an array of $\mathrm{N}$ filters that processes $\mathrm{N}$ input signals to give $\mathrm{N}$ outputs. The filter bank used at the transmitter side is called synthesis filter bank and the filter bank used in receiver side is called analysis filter bank. Filter bank multicarrier applies filtering on a persubcarrier foundation [4]. The plus point of this technique is that without using the $\mathrm{CP}$, it can give an efficient and better performance than conventional OFDM. With such attributes, it is considered as one of the most capable modulation technique for $5 \mathrm{G}$. This technique employs a bank of filters and the data in parallel pass through each filter. In FMBC offsetQAM is applied to comply with the factual orthogonality rule with the result FBMC exhibits nonorthogonality in the complex domain. OFDM with offset Quadrature amplitude modulation (OFDM/OQAM) is the most common FBMC technique. This filter method can be implemented in two ways - in the frequency domain, or in the time domain.

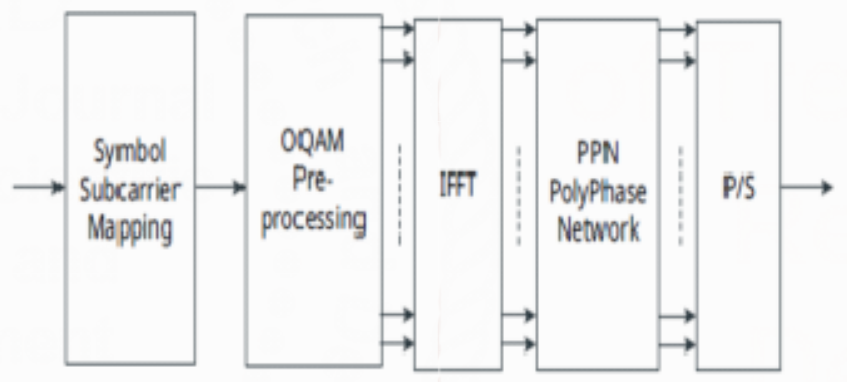

Figure 4: FBMC modulation block

To fix the iFFT length to the same total sub-carrier number, time domain processing method is suitable and Poly Phase Network (PPN) is used [5].FBMC using this narrowband filtering has greatly improved

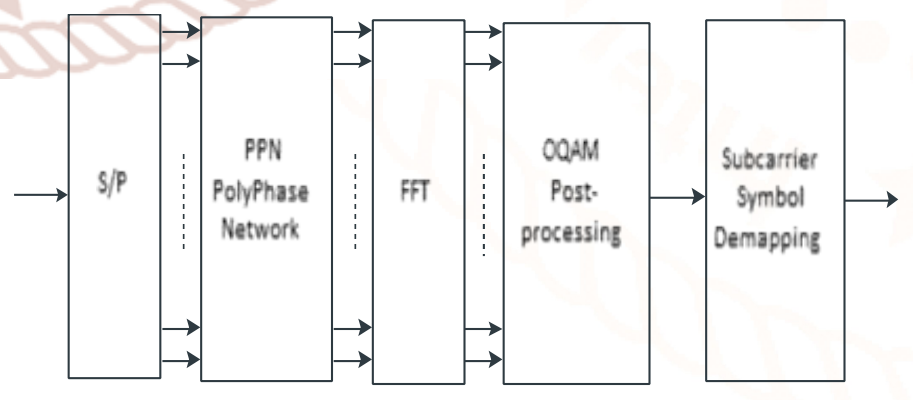

Figure 5: FBMC Demodulation Block

OOB Characteristics on the other hand, the number of filter taps required to improve the characteristics is about four times the total sub-carrier number, creating a four times processing latency in a PPN 
configuration. Accordingly, although FBMC is problem-free for bit pipe communications such as video streaming, it has lower transmission efficiency for short packets.

\section{UFMC}

Universal filtered multi-carrier (UFMC) is a waveform for future wireless systems. It is also known as unified filtered OFDM (UF-OFDM). UFMC is the method that combines the advantages of orthogonality OFDM and filter bank in FBMC.FBMC is problem-free for bit pipe communications such as video streaming, it has lower transmission efficiency for short packets .Instead of filtering each carrier like in FBMC, we have to filter a block of carriers called sub-band [4],[6]. Each sub band contains a number of carriers; filter length will be depending upon the width of sub-band. In the UFMC system, the complex symbols are generated from the base - band modulator. UFMC divides the band assigned for a user in smaller sub-bands, and after filtering them separately; they are summed and sent through the channel. Due to this filter tails, UFMC uses a null transmission or known as zero padding in front of each symbol, in order to cope with both, the delay spread of the channel and filter. UF-OFDM is a method for improving OOB characteristics by filtering each block. The mapped signal is allocated to a predetermine number of blocks. To overcome the problems of the ofdm we should have a new waveform for replacing OFDM in 5G physical layer.

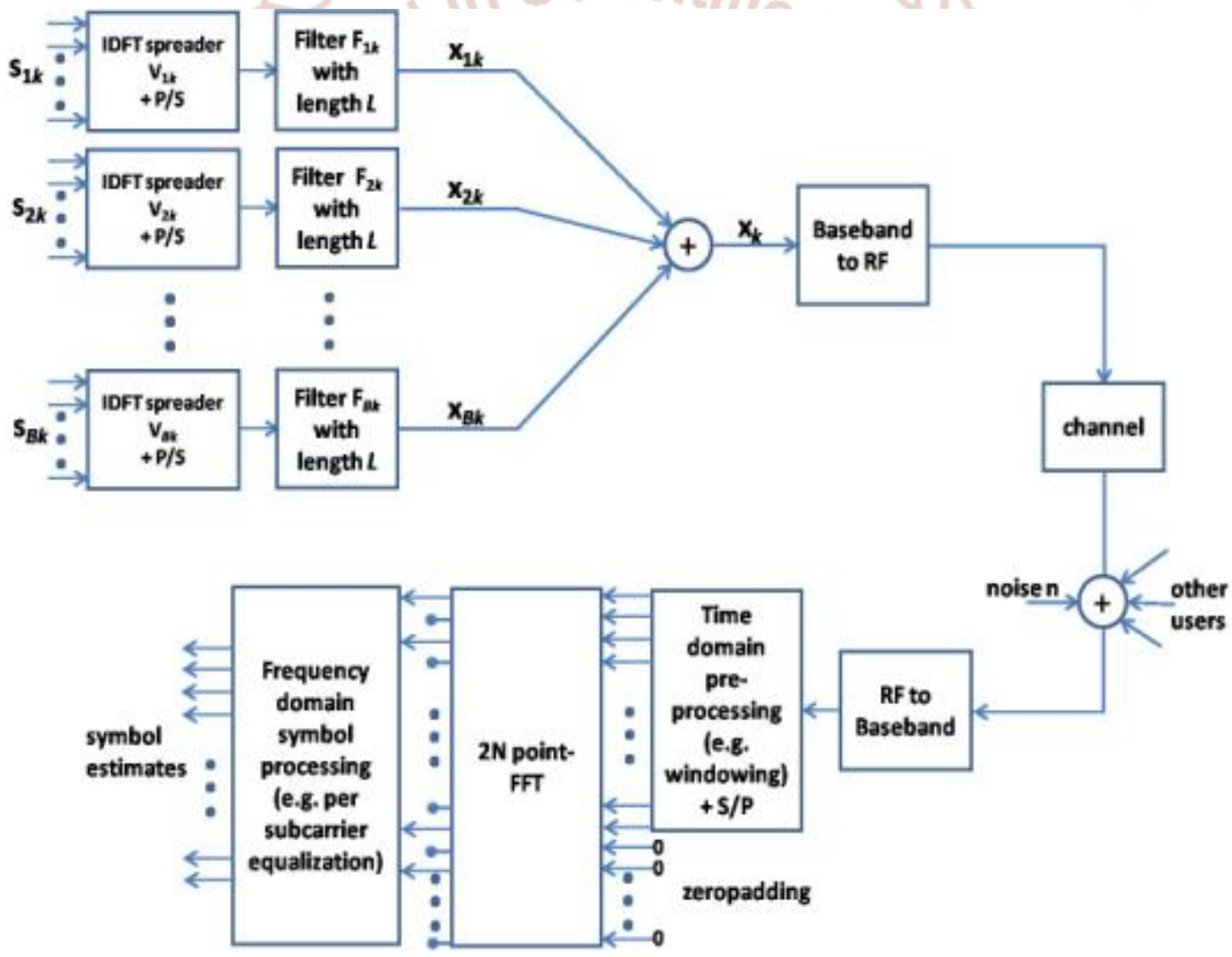

Figure 6: Block Diagram of UFMC Transceiver [7]

The new waveform should achieve the asynchronous reception and transmission, non - orthogonal waveforms for better spectral efficiency and low latency. The filter using in UFMC is DolphChebyshev of length ' $L$ '.

\section{Simulation Results}

Table 1: Simulation Parameter

\begin{tabular}{|l|l|}
\hline 1) Tool & Matlab \\
\hline 2) Modulation & Qpsk \\
\hline 3)FFT Size & 612 \\
\hline 4)CP Length & $16 \mathrm{bit}$ \\
\hline 5)Frequency & $4 \mathrm{GHz}$ \\
\hline 6)SNR & $1 \mathrm{db}$ to $25 \mathrm{db}$ \\
\hline 7)Channel & AWGN \\
\hline
\end{tabular}


Figure 7 shows BER performances of each system.

The analysis compares bit error rate (BER) vs SNR graph of FBMC and UFMC with OFDM modulation. FBMC offers ways to overcome the known limitations of OFDM of reduced spectral efficiency and strict synchronization requirements. The benefits have led it to being measured as one of the modulation techniques for $5 \mathrm{G}$ communication structures. UFMC technique shows the least bit error rate as compared to the above methods and it is the best waveform contenders for $5 \mathrm{G}$ applications [8].

Figure 8 shows the evaluation of FBMC and UFMC with OFDM on the basis of spectral efficiency vs number of users. It has been shown that UFMC exhibits higher spectral efficiency. It is multi-carrier transmission scheme in order to overcome the ICI problem and improve the system performance [9]. Due to the use of cyclic prefix in OFDM, $10 \%$ of the bits are repeated, where as in UFMC, cyclic prefix is not used. This increases spectral efficiency. In UFMC, total bandwidth is divided into sub-bands. As number of subcarriers adding up in phase will be fewer in UFMC, the maximum power decreases.

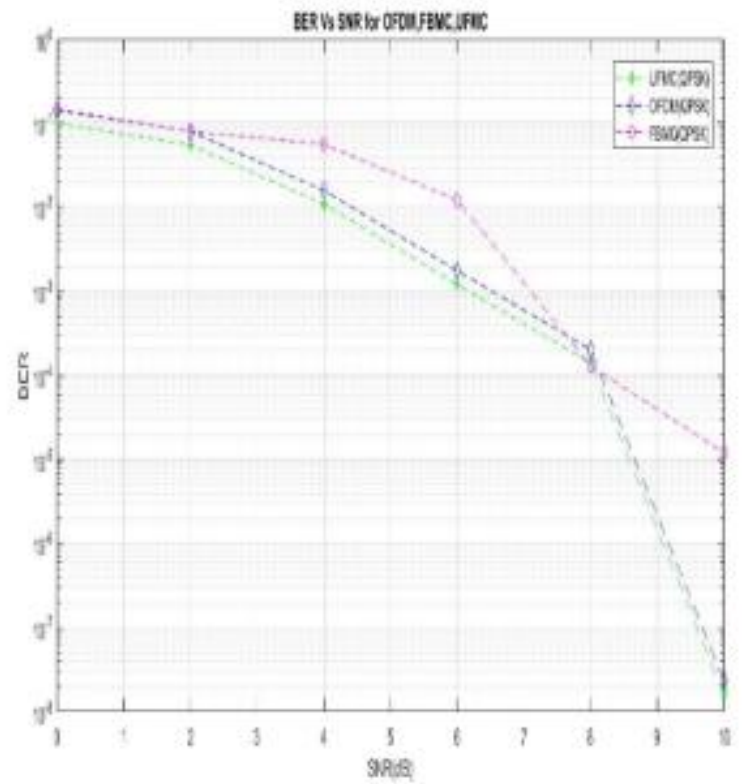

Figure 7: Graph of BER vs SNR for OFDM,FBMC and UFMC

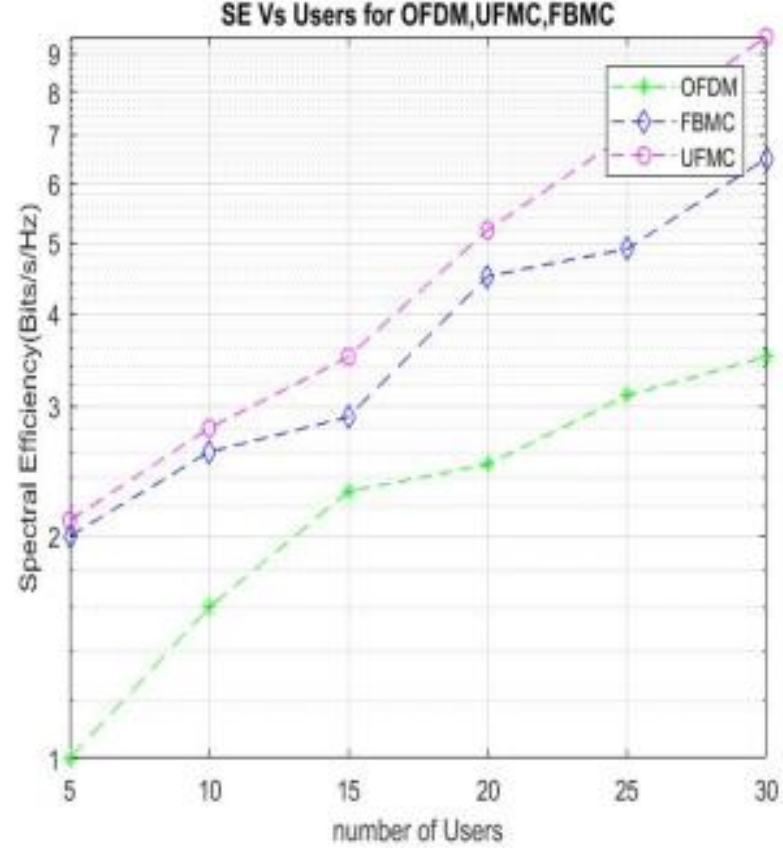

Figure 8: Graph of SE vS Users for UFDM,FBMC and UFMC.

\section{Conclusion}

Mobile communication requirements are increasing with the upcoming $5 \mathrm{G}$ applications such as Tactile Internet, IoT and Fragmented Spectrum. In this paper, we have focused on spectrum efficiency and BER performance levaluation of OFDM, FBMC, and UFMC.FBMC provides filtering of each subcarrier, which requires very long filter length and it prevents ICI. Although FBMC has several advantages as compared to the current OFDM of LTE, it is still not the optimal solution. With UFMC the objective is to merge the advantages of FBMC and filtered OFDM and get rid of some disadvantages of them. It is based on the idea of filtering a block of sub carriers [8],[10]. Like FBMC, UFMC is robust to multi-user interference, exhibits higher spectral efficiency and better suited to fragmented spectrum than OFDM.

\section{References}

1. W. Chin, F. Zhong, R. Haines, "Emerging technologies and research challenges for $5 \mathrm{G}$ wireless networks," IEEE Wireless Commun., vol. 21 , no.

2. pp. 106-112, Apr 2014. 2. J. Andrews, S. Buzzi, W. Choi, S. Hanly, A. Lozano, A. Soong, J. Zhang, "What will 5G be?" IEEE J. Select. Areas Commun., vol. 32, no. 6, pp. 1065-1082, Jun 2014.

3. A. S, ahin, I. G"uvenc, H. Arslan, "A survey on multi-carrier communications: Prototype filters, 
lattice structures, and implementation aspects, "IEEE Commun. Surveys Tutorials, vol. 16, no. 3, pp. 1312- 1338, Aug 2014.

4. P. Banelli, S. Buzzi, G. Colavolpe, A. Modenini, F. Rusek, A. Ugolini, "Modulation formats and waveforms for $5 \mathrm{G}$ net-works: Who will be the heir of OFDM?: An overview of alternative modulation schemes for improved spectral efficiency," IEEE Signal Process. Mag., vol. 31, no. 6, pp. 80-93, Nov 2014.

5. 3GPP, “Technical specification 36.212," Tech. Rep., Jun 2015, v12.5.0.

6. https://www.metis2020.com Mobile and Wireless Communications Enablers for the 2020 Information society.
7. http://www.5gnow.eu 5th Generation NonOrthogonal wave forms for asynchronous Signalling

8. A. Loulou, M. Renfors, "Enhanced OFDM for fragmented spectrum use in $5 \mathrm{G}$ systems", Trans. Emerging Tel. Tech., vol. 26, pp. 31-45, 20159) F. Schaich, T. Wild, Y. Chen, "Waveform contenders for $5 \mathrm{G}$ suitability for short packet and low latency transmissions, IEEE Vech. Technology Conference Spring, pp. 1-5, Apr 2014.

9. http://www.ict-phydyas.org Physical layer for dynamic spectrum access and cognitive radio.

10. 10.G. Matz, H. Bölcskei, F. Hlawatsch, "TimeFrequency Foundations of Communications," IEEE Signal 